\title{
Time-dependent response of both ventricles after septal ablation: Implications for biventricular support after left ventricular assist device placement
}

\author{
James Mau, BSc, MB, BS, ${ }^{\text {a }}$ Stuart Menzie, MB, BS, FRACS, ${ }^{a}$ Michael Ward, MB, BS, PhD, FRACP, ${ }^{b}$
} Henning Bundgaard, MD, PhD, and Stephen Hunyor, MB, BS, MD, MTM, FRACP, FACC ${ }^{\mathrm{a}}$

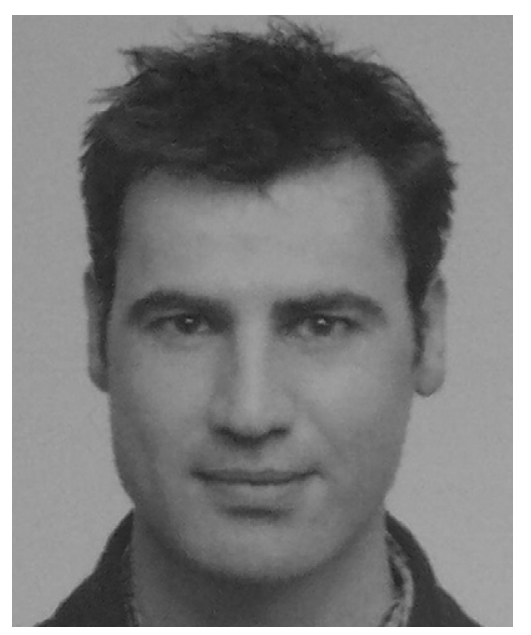

Dr Mau
From the Cardiac Technology Centre, Kolling Institute, ${ }^{\mathrm{a}}$ and Department of Cardiology ${ }^{\mathrm{b}}$ at Royal North Shore Hospital, University of Sydney, Australia.

Supported by the National Health and Medical Research Council of Australia (Grant 262031) and the North Shore Heart Research Foundation (Grants 16-04/05 and 19-01/02). James Mau is a Dora Lush Scholar of the National Health and Medical Research Council.

Received for publication Dec 20, 2006; revisions received Feb 23, 2007; accepted for publication March 26, 2007.

Reprint requests: James Mau, BSc, MB, BS, Cardiac Technology Centre, Block 4, Level 3, Royal North Shore Hospital, Pacific Hwy, St Leonards, Sydney, NSW, Australia 2065 (E-mail: jmau@med.usyd. edu.au).

J Thorac Cardiovasc Surg 2007;134:579-86 $0022-5223 / \$ 32.00$

Copyright (C) 2007 by The American Association for Thoracic Surgery

doi:10.1016/j.jtcvs.2007.03.048
Objectives: An ovine model of septal ablation was studied to elucidate the mechanisms involved in right ventricular failure when commencing left ventricular mechanical assistance. The disruption of ventricular interdependence after acute and chronic septal injury was examined.

Methods: Twelve sheep underwent percutaneous transluminal septal myocardial ablation using $0.6 \mathrm{~mL}$ ethanol. Twelve other sheep underwent a sham procedure. Left ventricular and right ventricular pressure and volume (conductance) response 15 minutes and 4 weeks postinjury were measured. Ultrasonic crystals measured chamber dimensions and wall movement. Areas at risk and infarct zones were quantified.

Results: Compared with sham, ablation chronically reduced systolic interventricular septal thickening $(18.4 \% \pm 5.8 \%$ vs $7.3 \% \pm 3.1 \% ; P<.001)$ and acutely increased right ventricular ejection fraction $(37.6 \% \pm 8.5 \%$ vs $69.9 \% \pm 7.2 \% ; P<.001)$, preload recruitable stroke work $\left(42.0 \pm 4.4 \mathrm{erg} \cdot 10^{3}\right.$ vs $48.7 \pm 2.0 \mathrm{erg} \cdot 10^{3}, P<$ $.001)$, end-systolic elastance (1.03 $\pm 0.19 \mathrm{~mm} \mathrm{Hg} \mathrm{mL}^{-1}$ vs $1.31 \pm 0.18 \mathrm{~mm} \mathrm{Hg}$ $\left.\mathrm{mL}^{-1} ; P<.001\right)$, and Tau $(24.9 \pm 3.8 \mathrm{~ms}$ vs $29.6 \pm 8.2 \mathrm{~ms} ; P<.001)$. In contrast, for left ventricular ejection fraction $(55.5 \% \pm 5.9 \%$ vs $38.9 \% \pm 7.7 \% ; P<.001)$, preload recruitable stroke work $(85.9 \pm 10.6 \mathrm{~mm} \mathrm{Hg}$ vs $66.5 \pm 9.6 \mathrm{~mm} \mathrm{Hg} ; P<$ $.001)$ and elastance $\left(2.13 \pm 0.51 \mathrm{~mm} \mathrm{Hg} \mathrm{mL}^{-1}\right.$ vs $1.81 \pm 0.44 \mathrm{~mm} \mathrm{Hg} \mathrm{mL}^{-1} ; P<$ $.001)$ were reduced, but Tau increased $(22.0 \pm 3.5 \mathrm{~ms}$ vs $28.9 \pm 5.8 \mathrm{~ms} ; P<.001)$ and remained elevated at 4 weeks compared with sham. The area at risk was the same between groups, and injury was limited to the septum $(17.2 \% \pm 2.7 \%$ vs $2.9 \% \pm$ $5.8 \% ; P<.001)$.

Conclusions: Acute and chronic hemodynamic responses are distinctly different after septal injury; the acute response demonstrates a paradoxical motion. Resolution of this motion at 4 weeks is suggestive of reduced septal compliance and buttressing. Ventricular interactions after placement of a left ventricular assist device will vary depending on the injury duration.

I nterest in right ventricular (RV) failure after acute mechanical left ventricular (LV) unloading for end-stage heart failure has followed the landmark REMATCH study ${ }^{1}$ and the recent report by Birks and colleagues, ${ }^{2}$ with implications for LV assist devices as destination therapy. Perioperative RV failure affects up to $25 \%$ of recipients and results in inadequate device filling and compromised multiple organ function. ${ }^{3}$ To avoid prolonged use of inotropes that can further impair RV function, severely ill recipients often require implantation of an additional right-sided support device. ${ }^{4}$

Disruption of septal position and function because of device-induced chamber attenuation has been demonstrated to contribute to altered RV contractility, ${ }^{5}$ load, ${ }^{6}$ 


$$
\begin{aligned}
& \text { Abbreviations and Acronyms } \\
& \begin{aligned}
\text { CVP }= & \text { central venous pressure } \\
\text { Ees }= & \text { slope of end-systolic pressure-volume } \\
& \text { relationship } \\
\mathrm{EF}= & \text { ejection fraction } \\
\mathrm{LV}= & \text { left ventricular } \\
\mathrm{PRSW}= & \text { preload recruitable stroke work } \\
\mathrm{RV} & =\text { right ventricular }
\end{aligned}
\end{aligned}
$$

and compliance ${ }^{7}$ by interfering with ventricular interdependence. Although it has been proposed that worsened RV decompensation results from coexisting septal damage, investigations have been confined to reversible septal injury. ${ }^{8}$ Percutaneous transluminal septal myocardial ablation with ethanol, ${ }^{9}$ developed as a treatment for hypertrophic obstructive cardiomyopathy, ${ }^{10}$ has enabled detailed study of ventricular interaction after both acute and chronic septal damage.

This study describes the acute and chronic biventricular response to ethanol ablation in a sheep model, using both steady-state load-independent and in-series measures. The findings aim to provide guidance on the likely need for RV support when LV mechanical assist device implantation is contemplated.

\section{Materials and Methods \\ Anesthesia}

Thirty-three Border Leicester cross sheep $(60.6 \pm 8.2 \mathrm{~kg})$ were approved for use by the Institutional Animal Care and Ethics Committee and received humane care in compliance with the "Guide for the Care and Use of Laboratory Animals," prepared by the Institute of Laboratory Animals Resources, National Research Council (1996). Animals were acclimatized for at least 2 weeks before random assignment into septal ablation or sham experimental groups. Anesthesia was induced by alfaxalone $(5-10 \mathrm{mg} / \mathrm{kg}$ ) and maintained with $50 \%$ oxygen, $45 \% / 50 \%$ nitrous oxide, and approximately $2 \%$ isoflurane. According to regular blood gas measurements, physiologic ventilation and oxygenation were ensured.

\section{Septal Myocardial Ablation}

The right jugular vein was cannulated, and a Capsure SP-4524 59-cm bipolar pacemaker lead (Medtronic; Minneapolis, Minn) was secured to the RV endocardial apex fluoroscopically and connected to a Sigma SR 300 implantable single-chamber bipolar pacemaker (Medtronic) programmed to a hysteresis of 50 beats/ min and pacing rate of 80 paces/min. A $2.0 \times 9-\mathrm{mm}$ Maverick SoftLEAP over-the-wire balloon catheter (Boston Scientific, Natick, Mass) was mounted on a standard 0.3556-mm angio wire, guided into the main septal perforating artery and positioned fluoroscopically with proximal clearance of the left anterior descending coronary artery. Correct positioning of the radio-opaque markers within the main septal perforator was considered completion of a sham procedure. A repeat angiogram verified patency. Active ablation involved balloon inflation to 8 atmospheres pressure followed by an immediate bolus injection of $0.6 \mathrm{~mL}$ of ethanol (99.6\%). Repeat angiography at 10 minutes in this group verified postablative main septal perforator closure and left anterior descending patency (Figure 1).

\section{Surgical Instrumentation and Hemodynamic Measurements}

Acute and chronic closed chest hemodynamic instrumentation involved cannulation of the left jugular vein and carotid artery with placement of a $6 \mathrm{~F}$ pressure-volume conductance catheter (Cardiodynamics; Rijinsberg, The Netherlands) and a 5F Millar cathetertip pressure transducer into each ventricle, and a transvenous $6 \mathrm{~F}$ Swan-Ganz catheter into the pulmonary artery. A 36F Fogarty catheter was positioned in the inferior vena cava for transient vena-caval occlusion.

Open chest ventricular dimension measurements involved placement of 2 pairs of 2-mm ultrasonic transducers (Sonometrics Corporation; London, Ontario) on the RV and LV free wall epicardium and endocardium and another pair on the RV and LV surface of the septum at the midpapillary level. A left thoracotomy with removal of the fifth rib provided access to the heart and great vessels, and the pericardium was incised along its left anterior edge from apex to base. All transducers were placed in an equatorial plane across the cardiac short axis, and their position was verified at postmortem. Ultrasonic flow probes (16 and $20 \mathrm{~mm}$ ) (Transonic Systems Inc, Ithaca, NY) were positioned around the aorta and pulmonary artery, respectively, and the incised pericardium was repositioned and resutured around the instrumented heart.

\section{Hemodynamic Assessment}

Data were collected at baseline and 15 minutes and 4 weeks after injury. Measurements were taken after at least 30 minutes of stable anesthesia at end expiration and included the following: biventricular measurements of (1) preload recruitable stroke work (PRSW), (2) end-systolic pressure volume relationship (Ees), (3) ejection fraction (EF), (4) Tau (early diastolic relaxation, calculated as the time from $\mathrm{dP} / \mathrm{dt}_{\text {min }}$ until pressure reached $\left.1 / 2 \mathrm{dP} / \mathrm{dt}_{\text {min }}\right),(5)$ central venous pressure (CVP), (6) RV peak systolic and end-diastolic pressure, and (7) pulmonary wedge pressure as previously described. ${ }^{11}$ Conductance measurements were calibrated against stroke volume values obtained from the respective flow probes.

\section{Infarct Size Determination}

At the completion of chronic hemodynamic measurements, hearts were excised and washed in normal saline before the left and right main coronary arteries and the main septal perforating artery were cannulated; $1 \%$ triphenyltetrazolium was used to selectively perfuse the main septal perforator at $100 \mathrm{~mm} \mathrm{Hg}$ to determine infarct size, ${ }^{12}$ with simultaneous perfusion of $10 \%$ methylene blue into the left and right coronary systems at equivalent pressures. Weights of the ventricles, areas at risk, and ablated zones were quantified by volume displacement and digital photo planimetry. Location of septal ultrasonic crystals in the central region of septal ablation was verified.

\section{Data Analysis}

Steady-state hemodynamic parameters were calculated as the average of 8 beats (including LV and RV end-systolic and enddiastolic pressures and volumes). PRSW relationship was calculated from the plot of stroke work against its loading end-diastolic 

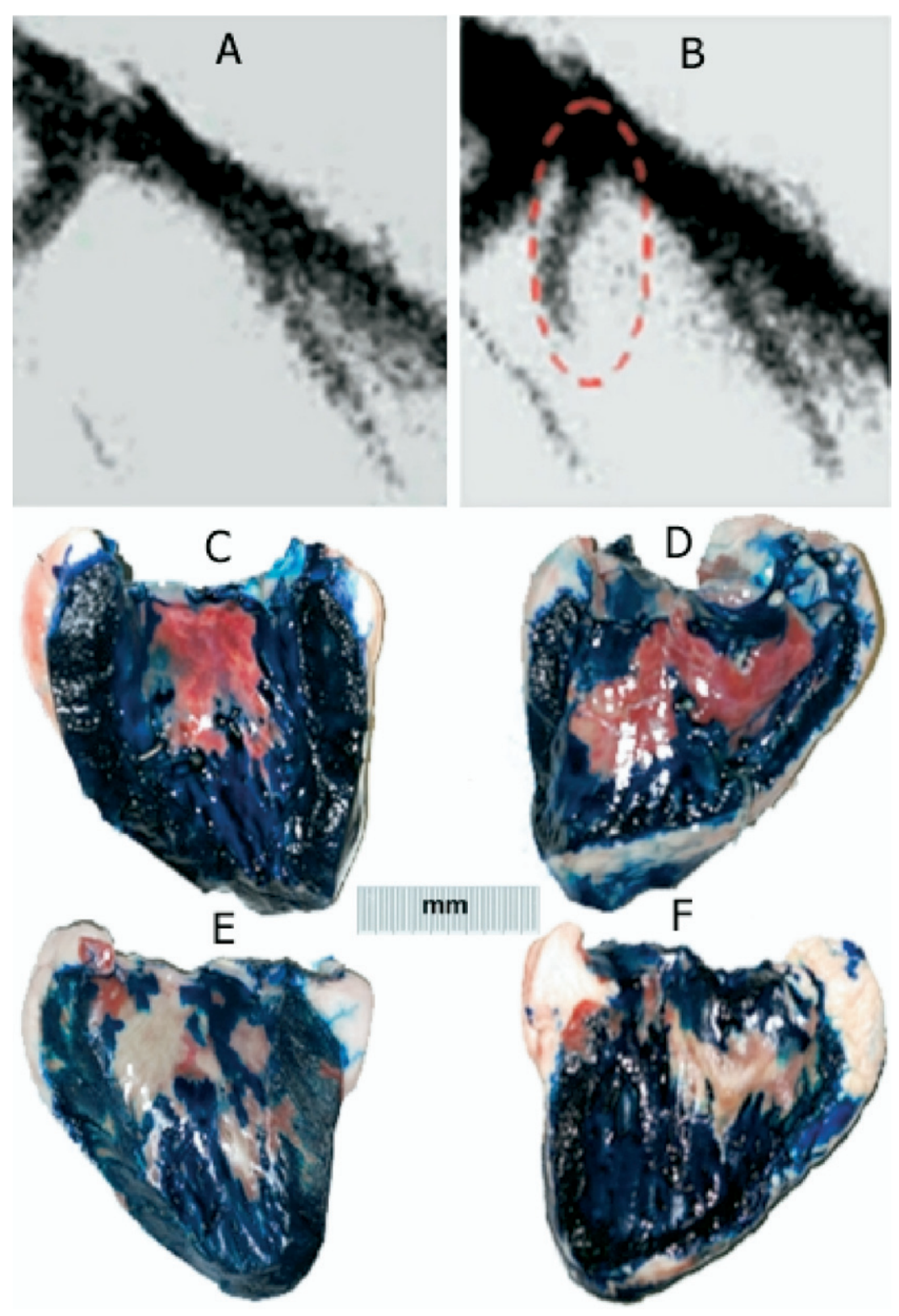

Figure 1. A, Postprocedural angiogram showing acute closure of the main septal perforating artery, which is circled in preprocedural angiogram (B). Sham (C, D) and ablation (E, F) postmortem hearts after differential staining with $1 \%$ triphenyltetrazolium solution and methylene blue dye at 4 weeks. Extensive fibrosis (white) is seen on the left (E) and right (F) septal surfaces after ablation compared with corresponding undamaged, triphenyltetrazolium-stained red areas (C, D) after a sham procedure. volume, and slope of the Ees was derived as previously described. ${ }^{11}$ End-diastolic and end-systolic crystal-derived dimensions were also analyzed as the average of 8 cardiac cycles during steady-state conditions. End diastole was defined as the time of peak electrocardiogram R wave, and RV and LV end systole were defined as the point of zero pulmonary or aortic flows, respectively. Fractional systolic change in wall thickness was calculated from sonomicrometry-derived dimensions as (end-systolic-enddiastolic dimension)*100/end-diastolic dimension. Randomly coded digital photographs of infarct zones were analyzed by a blinded operator using Sigma Scan Image v2.03 (SPSS Inc, Chicago, Ill). Pacemaker interrogation provided a 4-week history of pacing need.

\section{Statistical Analysis}

The results are presented as means \pm standard deviation. For all parameters, comparisons between sham and ablation groups were calculated using a 2-tailed $t$ test for parametric distributions or, where data failed a normality test, a Mann-Whitney rank-sum test for nonparametric distributions. All statistical analyses were performed using SigmaStat Statistical Software v2.03 (SPSS Inc).

\section{Results}

A total of 24 of 33 animals enrolled were used for analysis, with 12 each in the ablation and sham groups. Four animals died of acute heart failure and/or irrecoverable arrhythmias after ethanol ablation; 3 animals died of acute heart failure during the terminal study; 1 animal was terminated during catheterization after embolization of a fractured angio wire fragment; and 1 animal was removed from analysis because of recurrent arrhythmias. 
Load-independent and In-series Hemodynamic Data Despite ethanol ablation, CVP, LV peak systolic pressure, $\mathrm{LV}$ and RV stroke volume, and cardiac output were not significantly different between sham and ablation groups. $\mathrm{LV}$ stroke volume averaged $57.1 \pm 6.6 \mathrm{~mL}, 54.9 \pm 11.0$ $\mathrm{mL}$, and $56.3 \pm 8.0 \mathrm{~mL}$ for the sham group and $53.3 \pm 10.3$ $\mathrm{mL}, 47.2 \pm 12.1 \mathrm{~mL}$, and $52.0 \pm 12.5 \mathrm{~mL}$ for the ablation group at baseline, 15 minutes, and 4 weeks $(P$ values $=$ $.229, .068, .290)$, respectively. RV stroke volume averaged $57.4 \pm 6.3 \mathrm{~mL}, 54.5 \pm 10.1 \mathrm{~mL}$, and $56.6 \pm 7.8 \mathrm{~mL}$ for the sham group and $53.5 \pm 9.0 \mathrm{~mL}, 48.3 \pm 13.0 \mathrm{~mL}$, and $54.1 \pm$ $13.7 \mathrm{~mL}$ for the ablation group at baseline, 15 minutes, and 4 weeks $(P$ values $=.173, .148, .559)$, respectively. $\mathrm{LV}$ cardiac output averaged $4.1 \pm 0.5 \mathrm{~L} / \mathrm{min}, 3.9 \pm 0.8 \mathrm{~L} / \mathrm{min}$, and $4.0 \pm 0.6 \mathrm{~L} / \mathrm{min}$ for the sham group and $3.7 \pm 0.4 \mathrm{~L} / \mathrm{min}$, $3.6 \pm 0.4 \mathrm{~L} / \mathrm{min}$, and $3.6 \pm 0.4 \mathrm{~L} / \mathrm{min}$ for the ablation group at baseline, 15 minutes, and 4 weeks $(P$ values $=.074, .637$, $.105)$, respectively. $\mathrm{RV}$ cardiac output averaged $4.1 \pm 0.5$ $\mathrm{L} / \mathrm{min}, 3.9 \pm 0.7 \mathrm{~L} / \mathrm{min}$, and $4.0 \pm 0.6 \mathrm{~L} / \mathrm{min}$ for the sham group and $3.7 \pm 0.5 \mathrm{~L} / \mathrm{min}, 3.7 \pm 0.5 \mathrm{~L} / \mathrm{min}$, and $3.8 \pm 0.6$ $\mathrm{L} / \mathrm{min}$ for the ablation group at baseline, 15 minutes, and 4 weeks $(P$ values $=.800, .494, .193)$ respectively.

Although pulmonary wedge pressure and RV peak systolic pressures in the ablation and sham groups were not statistically different at 4 weeks $(P=.129, .904$, respectively), they were higher in the ablation group at 15 minutes after intervention compared with sham values $(P<.001$ and $P=.044$, respectively, Figure 2). EFs and pressure volume derivatives are illustrated in Figure 3. RV PRSW, Ees, and EF values were higher 15 minutes after intervention compared with sham values, whereas LV values were lower $(P<.001)$. There was no difference in PRSW, Ees, and EF values between groups at 4 weeks (RV: $P=.516, .669$, and .086 ; LV: $P=.730, .581$, and .349 , respectively). RV and LV Tau values were higher 15 minutes after intervention compared with sham and remained higher at 4 weeks. As shown in Figure 4, LV end-systolic and end-diastolic volumes were higher, and corresponding RV volumes were lower at 15 minutes in ablation animals compared with sham animals $(P<.001)$. Comparison of RV and LV summed end-diastolic and end-systolic volumes revealed that overall cardiac volume was maintained at 15 minutes and 4 weeks, with $P$ values of .664 and .060 for 15 minutes and .233 and .244 for 4-week comparisons, respectively (Figure 4).

\section{Septal Functional Analysis}

Chronic septal functional and dimensional data are summarized in Table 1. At 4 weeks, ablation reduced systolic septal thickening by more than half $(7.3 \% \pm 3.1 \%$ in ablation vs $18.4 \% \pm 5.8 \%$ in sham, $P<.001$ ), and RV and LV free wall thickening and biventricular lateral cardiac diameter were maintained. Pacemaker interrogation re-

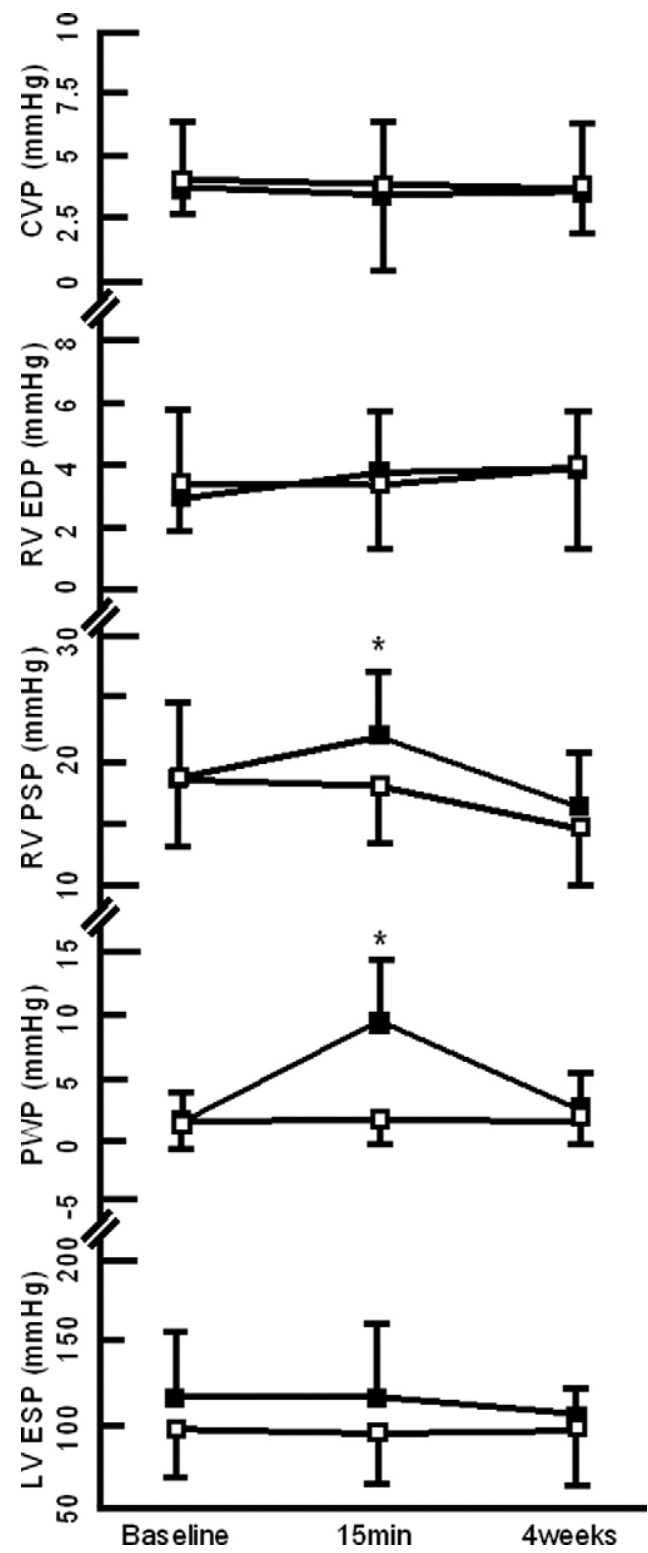

Figure 2. Effect of ethanol ablation (filled squares) on LV peak systolic pressure, RV peak systolic pressure, RV end-diastolic pressure, CVP, and pulmonary wedge pressure compared with sham (open squares) at baseline, 15 minutes after ethanol ablation, and 4 weeks (mean \pm standard deviation [SD]; ${ }^{*} \boldsymbol{P}<.001$ ). $L V$, Left ventricular; $R V$, right ventricular; $E S P$, end-systolic pressure; $P W P$, pulmonary wedge pressure; $P S P$, peak systolic pressure; EDP, end-diastolic pressure; CVP, central venous pressure.

vealed an increased pacing requirement in ablation animals $(24.4 \% \pm 10.9 \%$ vs $2.6 \% \pm 2.5 \%$ for sham, $P<.001)$.

\section{Triphenyltetrazolium Histologic analysis}

A representative postmortem heart with differential triphenyltetrazolium and methylene blue staining is displayed in 

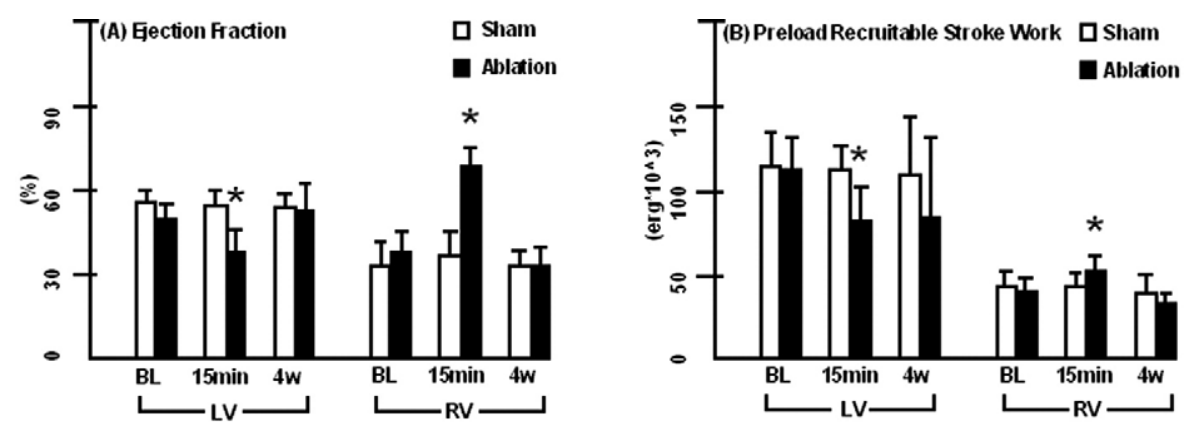

Figure 3. Comparison of LV and RV parameters in response to ethanol ablation. (A) EF, (B) PRSW, (C) end-systolic elastance, and (D) Tau (mean \pm SD; $\left.{ }^{*} P<.001\right)$. $L V$, Left ventricular; $R V$, right ventricular; $B L$, baseline.
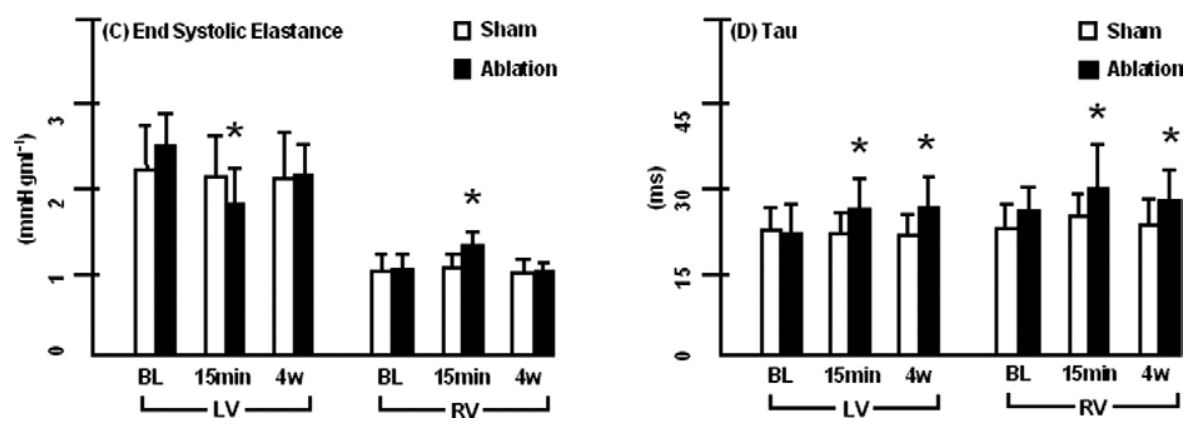

Figure 1. At 4 weeks, the ethanol ablation zone was confined to the septum, and there was no significant difference in the areas at risk between respective sham and ablation animals: $38.8 \% \pm 5.5 \%$ versus $37.0 \% \pm 5.7 \%, P=.429$; $41.7 \% \pm 11.8 \%$ versus $33.4 \% \pm 9.0 \%, P=.055$; and $26.0 \% \pm 9.6 \%$ vs $23.0 \% \pm 6.7, P=.365 \%$ of the LVseptal, RV-septal surface areas, and total percentage septal tissue by weight, respectively. The degree of infarcted tissue in ablation animals was markedly increased compared with sham animals $(P<.001$, Table 1$)$.

\section{Discussion}

The major findings of the present study are that ethanol ablation of the interventricular septum results in acute findings of paradoxical septal motion, LV dilatation, and RV shrinkage, but with maintained summed ventricular volume. Chronically, septal function is impaired. Also, there are contrasting acute and chronic hemodynamic responses.

\section{Systolic Heart Function}

Acutely, CVP and RV end-diastolic pressures were unaffected by ablation (Figure 2), whereas RV peak systolic pressure, Ees, PRSW, and EF were higher compared with sham values (Figure 3). This indicates no detriment to overall RV function. In contrast, in-series LV functional (pulmonary wedge pressures and EF) and load-independent indices were acutely impaired. Thus, in contrast with classic theory, ${ }^{13}$ a clear increase in wedge pressure after acute injury was identified. We postulate that this is caused by unmasking of an intact RV pressure/volume response during LV contractile failure, with maintenance of LV forward flow aided by elevated LV filling pressure. All systolic parameters returned to baseline by 4 weeks, indicating LV functional recovery (Figure 3). This is the first study to identify distinct differences between acute and chronic hemodynamic response after isolated septal injury. It also challenges and extends previous theories that sought to explain the phenomenon of RV failure after LV mechanical assistance using studies confined to acute, reversible ischemic models. ${ }^{8}$ Here we demonstrate that the chronic response to septal injury is distinctly different.

\section{Diastolic Heart Function}

Disruption of diastolic function resulting from myocardial injury is conditional on the specific pathology present and the resulting cellular response, including the action of matrix metalloproteinases and their tissue inhibitors. ${ }^{14}$ Reduction in tissue inhibitor of metalloproteinase- 4 appears to be characteristic of ethanol ablation. ${ }^{15} \mathrm{We}$ have demonstrated that ethanol ablation acutely prolongs left and $\mathrm{RV}$ relaxation times (Tau), which remain abnormal at 4 weeks (Figure 3). This is supported by other studies that revealed delayed RV early diastolic relaxation in response to acute septal ${ }^{16}$ and $\mathrm{LV}^{17}$ and $\mathrm{RV}^{18}$ free wall ischemia, as well as in the chronic setting after $\mathrm{LV}^{19}$ and $\mathrm{RV}^{20}$ infarction. This persistence of impaired diastolic relaxation after 4 weeks indicates a change that mirrors the reduction in tissue inhibitor of metalloproteinase-4. 


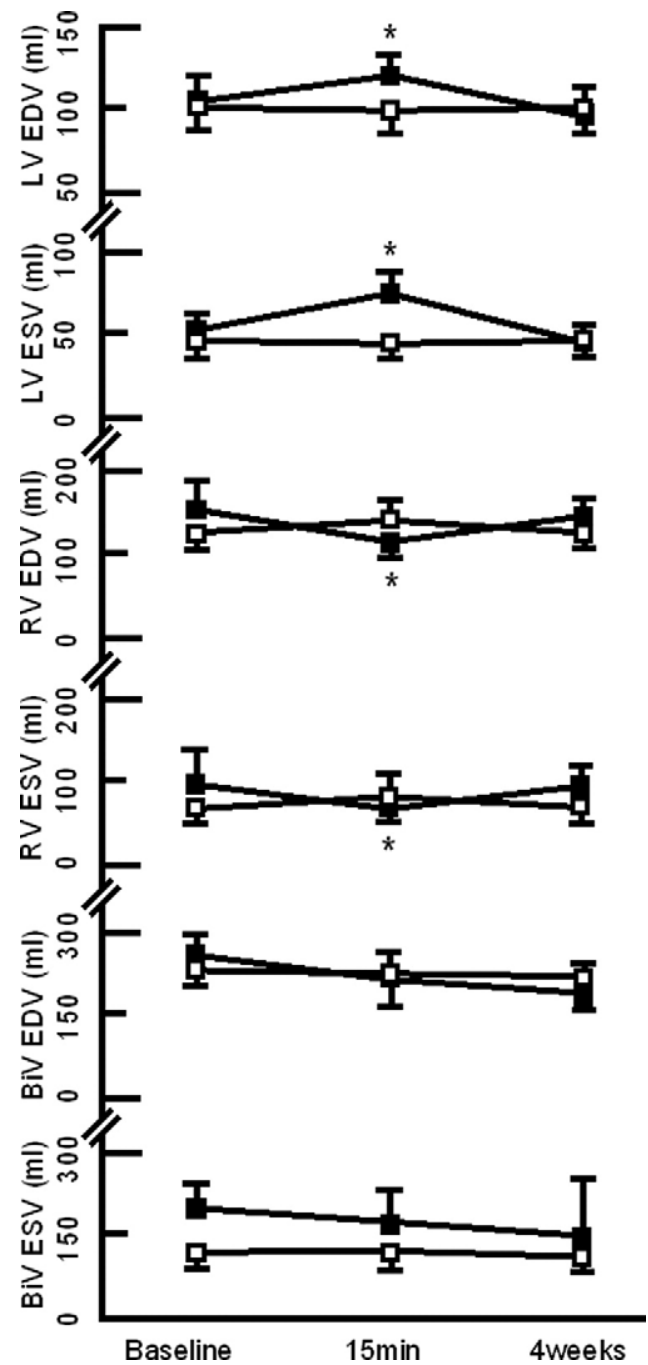

Figure 4. Effect of ethanol ablation (filled squares) on LV enddiastolic and systolic volumes, RV end-diastolic and systolic volumes, and biventricular (summed LV and RV) end-diastolic and systolic volumes compared with sham (open squares) at baseline, 15 minutes after ethanol ablation, and 4 weeks (mean \pm SD; ${ }^{*} P<$ .001). There is divergence between RV and LV volumes acutely after ablation, compared with sham response. In contrast, biventricular volumes remain stable. Acute changes in volume seen in ablation animals return to sham levels after 4 weeks (mean \pm SD; ${ }^{*} P<$.001). BiV, Biventricular; $R V$, right ventricular; $L V$, left ventricular; $E S V$, end-systolic volume; $E D V$, end-diastolic volume.

Ventricular Interdependence and Geometry

The role of the septum in maintaining LV and RV interdependence mechanisms is paramount. ${ }^{21,22}$ Physiologic pressure gradients are governed by active septal myocardial sarcomeric mechanisms with the end-diastolic relational position of the septum between the ventricles constituting a form of "septal preload." ${ }^{23}$ Removal of active septal systole
TABLE 1. Systolic septal thickening of the free walls and septum, triphenyltetrazolium staining of the septum, and lateral geometric changes in ablation and sham animals

\begin{tabular}{lrrr}
\hline & \multicolumn{1}{c}{ Sham } & Ablation & $\boldsymbol{P}$ value \\
\hline \% RVFW sst & $4.4 \pm 2.4$ & $4.8 \pm 2.7$ & .903 \\
\% Septal sst & $18.4 \pm 5.8$ & $7.3 \pm 3.1$ & $<.001$ \\
\% LVFW sst & $16.6 \pm 4.2$ & $14.7 \pm 3.1$ & .234 \\
$\begin{array}{l}\text { \% Lateral cardiac diameter } \\
\quad 20.2 \pm 3.9\end{array}$ & $21.7 \pm 4.1$ & .293 \\
$\quad$ change & & & \\
\% Pacing & $2.6 \pm 2.5$ & $24.4 \pm 10.9$ & $<.001$ \\
\% Infarct of RV-septal & $2.1 \pm 1.7$ & $19.0 \pm 6.1$ & $<.001$ \\
$\quad$ area & & & \\
\% Infarct of LV-septal area & $2.6 \pm 1.5$ & $29.5 \pm 5.1$ & $<.001$ \\
\% Infarct of total septal & $2.9 \pm 5.8$ & $17.2 \pm 2.7$ & $<.001$ \\
$\quad$ weight & & & \\
\end{tabular}

sst, Systolic septal thickening; $R V$, right ventricle; $L V$, left ventricle; $R V F W$, right ventricular free wall; $L V F W$, left ventricular free wall; $R V$-septal, right ventricle facing surface of the septum; LV-septal, left ventricle facing surface of the septum.

under conditions of unaltered myocardial compliance, such as that induced by ischemia or paralysis, results in paradoxical motion of the septum into the RV at points in the cardiac cycle where LV pressure dominates. Associated sarcomeric lengthening continues until its maximal length is reached and passive restraint prevents further RV-vectored motion. $^{22}$ Thus, according to the law of conservation of mass that describes associated increases in surface area of tissue under compression, transeptal pressure induces sarcomeric stretching and septal surface area expansion. Eventually, at maximal or close to maximal sarcomeric length, septal position becomes entirely subject to fluctuating pressure gradients and oscillates paradoxically throughout the cardiac cycle.

Because of the asymmetric RV shape and its tendency to alter with loading, we used the conductance method to avoid geometric assumptions. Compared with the tricuspid axis, the pulmonary axis provides better RV volumetric data and avoids the risk of right atrial sampling during diastolic tricuspid valve descent. In this study, a transvenous tricuspid approach was used to avoid an open chest procedure; however, conductance-derived stroke volume values were calibrated with a pulmonary flow probe. The conductance pattern after ablation reveals maintenance of stroke volume in both ventricles with an immediate reduction in LV EF and mirrored increases in RV EF (Figure 3). By 4 weeks, all acutely altered geometry returns to baseline values. $\mathrm{LV}$ and $\mathrm{RV}$ end-systolic and end-diastolic volume analysis reveals unaltered summed LV and RV (biventricular) volume and equal degrees of LV dilatation and RV shrinkage (Figure 4). Thus, the exaggerated septal motion in systole leads to an increase in RV EF and a decrease in RV end-systolic volume. In turn, this sets up subsequent diastole, with de- 
creased RV volume improving filling potential of the LV. The net effect is a minimal change to cardiac output and overall heart volume, suggesting either that physiologic septal movement in unison with the LV free wall is a redundant action or that additional factors are in operation to compensate for the offset hemodynamic performance induced by septal injury. Resolution of this motion after 4 weeks is suggestive of reduced septal compliance and buttressing.

\section{Validation of Septal Dysfunction}

Four weeks after ablation, our study showed a pattern of wall movement and dimensions similar to previous studies of acute septal ${ }^{8}$ and LV free wall ischemic injury. ${ }^{24}$ However, in contrast with sonomicrometric studies after anteroseptal infarction and temporary septal ischemia, ${ }^{25}$ we did not observe paradoxical septal thinning. Thus, either there is residual sarcomeric function to counteract passive thinning or there is chronic compliance change that passively resists altered transeptal pressure gradients. It is proposed that the latter mimics an ischemic fibrotic pattern. ${ }^{22}$ In addition to functional disruption, a conduction block requiring a 10fold increase in temporary pacing was noted that resolved by 4 weeks (Table 1). Anatomic studies of the sheep conductive pathway have revealed a more robust and narrow cable-like bifascicular Purkinje system ${ }^{26}$ compared with the broader, more susceptible trifascicular pattern in humans. ${ }^{27}$ Thus, ready resolution of short-term atrioventricular nodal edema and swelling could explain our results. Prior human studies suggest that the need for interim pacing after septal ablation typically translates to chronic conductive pathway pathology. ${ }^{28}$

\section{Postmortem Findings}

Postmortem findings confirmed adequacy of ethanol volume used in our ablation animals, showing a 10-fold increase in the fibrotic area as a proportion of area at risk. Figure 1 illustrates that fibrosis was confined to a centralized mass of tissue inferior to the trigonum fibrosum, corresponding to the principal supply area of the main septal perforating artery. This pattern of septal damage is borne out by the sonomicrometric data demonstrating a decrease in septal thickening, consistent with ethanol ablation causing damage of a chronic nature and previous echocardiographic findings of postablative septal thinning at the midpapillary plane. ${ }^{29}$

\section{Conclusions}

We conclude that pathophysiologic changes after ethanol ablation comprise the following: (1) Atrioventricular nodal edema and transient heart block, (2) septal segmental chord lengthening with loss of active sarcomeric contraction and resulting paradoxical septal motion, (3) contrasting acute responses of RV and LV load-independent functional indi- ces, (4) larger LV and smaller RV volumetric windows of operation, (5) maintenance of in-series aggregate RV function with temporary strain affecting LV in-series redundancy, and finally, (6) normalization of disrupted systolic function by 4 weeks.

\section{Limitations}

$\mathrm{RV}$ and LV function were investigated in an experimental model in which study of intrinsic septal function was confined to the chronic phase after ablation to avoid successive open chest procedures. A logical extension of this study would involve the assessment of septal function before and directly after ethanol ablation.

\section{Implications}

Ventricular interactions and acute RV failure etiology that often follow LV assist device implantation for severe heart failure have been characterized. Manifestation of occult RV dysfunction, LV geometric alterations resulting from LV unloading-induced LV volume minimization, and simple in-series interactions have all been implicated. The presence of septal ischemia has been shown to accentuate these processes. ${ }^{8}$ We have identified clear differences in ventricular interactions and geometry between acute and chronic septal injury under closed chest conditions. This extends the known pathophysiologic course after ethanol ablation and indicates that passive and active components of ventricular compliance and contractility are not interchangeable between ischemic and fibrotic pathologies. Thus, this study affords an avenue to further examine LV assist device interaction within a chronic pathologic setting. It calls into question the extrapolation of such interaction during acute septal injury as an explanation for abnormalities found in patients with a chronic component of septal disease.

We thank Yifei Huang, Kellie Tinworth, Gabrielle Gallagher, Bruce Williams, Ray Kearns, Wayne Roach, and Ms Cathy Mundy for technical assistance. Medtronic Inc supplied the pacemakers, and Chris McLure advised on their use.

\section{References}

1. Rose EA, Gelijns AC, Moskowitz AJ, Heitjan DF, Stevenson LW, Dembitsky W, et al. Long-term mechanical left ventricular assistance for end-stage heart failure: Randomised Evaluation of Mechanical Assistance for the Treatment of Congestive Heart Failure (REMATCH). N Engl J Med. 2001;345:1435-43.

2. Birks EJ, Tansley PD, Hardy J, George RS, Bowles CT, Burke M, et al. Left ventricular assist device and drug therapy for the reversal of heart failure. N Engl J Med. 2006;355:1873-84.

3. Furukawa K, Motomura T, Nose Y. Right ventricular failure after left ventricular assist device implantation: the need for an implantable right ventricular assist device. Artif Organs. 2005;29:369-77.

4. Goldstein DJ, Oz MC, Rose EA. Implantable left ventricular assist devices. N Engl J Med. 1998;339:1522-33.

5. Moon MR, Castro LJ, DeAnda A, Tomizawa Y, Daughters GT 2nd, Ingels NB Jr, et al. Right ventricular dynamics during left ventricular assistance in closed-chest dogs. Ann Thorac Surg. 1993;56:54-67. 
6. Morita S, Kormos RL, Mandarino WA, Eishi K, Kawai A, Gasior TA, et al. Right ventricular/arterial coupling in the patient with left ventricular assistance. Circulation. 1992;86:S316-25.

7. Elbeery JR, Owen CH, Savitt MA, Davis JW, Feneley MP, Rankin JS, et al. Effects of the left ventricular assist device on right ventricular function. J Thorac Cardiovasc Surg. 1990;99:809-16.

8. Daly RC, Chandrasekaran K, Cavarocchi NC, Tajik AJ, Schaff HV. Ischemia of the interventricular septum. A mechanism of right ventricular failure during mechanical left ventricular assist. J Thorac Cardiovasc Surg. 1992;103:1186-91.

9. Nielsen CD, Fernandes V, Spencer WH. Alcohol septal ablation for obstructive hypertrophic cardiomyopathy. Am Heart J. 2003;1:83-90.

10. Veselka J, Duchonova R, Prochazkova S, Homolova I, Palenickova J, Zemanek D, et al. The biphasic course of changes of left ventricular outflow gradient after alcohol septal ablation for hypertrophic obstructive cardiomyopathy. Kardiologia Polska. 2004;60:133-7.

11. Huang Y, Hunyor SN, Jiang L, Kawaguchi O, Shirota K, Ikeda Y, et al. Remodeling of the chronic severely failing ischemic sheep heart after coronary microembolization: functional, energetic, structural, and cellular responses. Am J Physiol Heart Circ Physiol. 2004;286:2141-50.

12. Fishbein MC, Meerbaum S, Rit J, Lando U, Kanmatsuse K, Mercier $\mathrm{JC}$, et al. Early phase acute myocardial infarct size quantification: validation of the triphenyl tetrazolium chloride tissue enzyme staining technique. Am Heart J. 1981;101:593-600.

13. Karliner JS, Ross JJ. Left ventricular performance after acute myocardial infarction. Prog Cardiovasc Dis. 1971;13:374-91.

14. Gallagher G, Jackson CJ, Hunyor SN. Myocardial extracellular matrix remodeling in ischemic heart failure. Front Biosci. 2007;12:1410-9.

15. Stroud RE, Deschamps AM, Lowry AS, Hardin AE, Mukherjee R, Lindsey ML, et al. Plasma monitoring of the myocardial specific tissue inhibitor of metalloproteinase-4 after alcohol septal ablation in hypertrophic obstructive cardiomyopathy. J Card Fail. 2005;11:124-30.

16. Sugihara H, Adachi H, Nakagawa T, Inagaki S, Kubota Y, Ebizawa T, et al. Right ventricular systolic and diastolic functions assessed by $81 \mathrm{mKr}$ scintigraphy and relation to ventricular septal ischemia. J Cardiol. 1987; 17:607-15.

17. Miyazaki S, Guth BD, Miura T, Indolfi C, Schulz R, Ross JJ. Changes of left ventricular diastolic function in exercising dogs without and with ischemia. Circulation. 1990;81:1058-70.
18. Pasipoularides AD, Shu M, Shah A, Glower DD. Right ventricular diastolic relaxation in conscious dog models of pressure overload, volume overload, and ischemia. J Thorac Cardiovasc Surg. 2002;124: 964-72.

19. Marsch SC, Dalmas S, Philbin DM, Wanigasekera VA, Ryder WA, Wong LS, et al. Post-ischemic diastolic dysfunction. J Cardiothorac Vasc Anesth. 1994;8:611-7.

20. Calvin JE. Right ventricular diastolic function after experimental right ventricular infarction: effects independent of the pericardium. Clin Invest Med. 1991;14:346-54.

21. Santamore WP, Dell'Italia LJ. Ventricular interdependence: significant left ventricular contributions to right ventricular systolic function. Prog Cardiovasc Dis. 1998;40:289-308.

22. Buckberg GD, Coghlan HC, Hoffman JI, Torrent-Guasp F. The structure and function of the helical heart and its buttress wrapping VII: critical importance of septum for right ventricular function. Semin Thorac Cardiovasc Surg. 2001;13:402-16.

23. Klima UP, Lee MY, Guerrero JL, Laraia PJ, Levine RA, Vlahakes GJ Determinants of maximal right ventricular function: role of septal shift. J Thorac Cardiovasc Surg. 2002;123:72-80.

24. Lekven J, Mjos OD, Kjekshus JK. Compensatory mechanisms during graded myocardial ischemia. Am J Cardiol. 1973;31:467-73.

25. Molaug M, Geiran O, Kiil F. Compensatory cardiac mechanisms evoked by septal ischemia in dogs. Am J Cardiol. 1983;51:201-6.

26. Ansari A, Ho S, Anderson RN. Distribution of the Purkinje fibres in the sheep. Anat Rec. 1999;254:92-7.

27. Janse MJ, Rosen MR. History of arrhythmias. Handb Exp Pharmacol. 2006:1-39.

28. Chen AA, Palacios IF, Mela T, Yoerger DM, Picard MH, Vlahakes G, et al. Acute predictors of subacute complete heart block after alcohol septal ablation for obstructive hypertrophic cardiomyopathy. Am J Cardiol. 2006;97:264-9.

29. Veselka J, Prochazkova S, Duchonova R, Bolomova-Homolova I, Palenickova J, Tesar D, et al. Alcohol septal ablation for hypertrophic obstructive cardiomyopathy: lower alcohol dose reduces size of infarction and has comparable hemodynamic and clinical outcome. Catheter Cardiovasc Interv. 2004;63:231-5. 